Revista Brasileira de Agricultura Irrigada v.13, nº.5, p. 3637 - 3645, 2019

ISSN 1982-7679 (On-line)

Fortaleza, CE, INOVAGRI - http://www.inovagri.org.br

DOI: $10.7127 /$ rbai.v13n5001122

Protocolo 1122.19 - 25/03/2020 Aprovado em 30/03/2020

\title{
ESTRESSE SALINO, COBERTURA MORTA E TURNO DE REGA NA CULTURA DO SORGO
}

Carla Ingryd Nojosa Lessa ${ }^{1}$, Ádila Cristina Nunes de Oliveira ${ }^{2}$, Clarissa Lima Magalhães ${ }^{3}$, José Thomas Machado de Sousa ${ }^{4}$, Geocleber Gomes de Sousa ${ }^{5}$

\begin{abstract}
RESUMO
O objetivo deste trabalho foi avaliar o efeito da irrigação com água de alta e baixa salinidade em diferentes turnos de rega e cobertura morta, na cultura do sorgo. $\mathrm{O}$ experimento foi conduzido na área experimental da Unidade de Produção de Mudas Auroras (UPMA) da Universidade da Integração Internacional da Lusofonia Afro-Brasileira (UNILAB), com o delineamento inteiramente casualizado, em arranjo fatorial $2 \times 2 \times 2$, sendo dois níveis de condutividade elétrica (A1 - água de abastecimento $0,8 \mathrm{dS} \mathrm{m}^{-1}$ e A2 - solução salina 4,0 dS m${ }^{-1}$ ), dois turnos de rega (T1 - turno de rega diário e T2 - turno de rega a cada 4 dias) e, dois tipos de cobertura ( $\mathrm{Cc}$ - com cobertura morta e Sc sem cobertura). Foram avaliadas as seguintes variáveis: área foliar (AF), diâmetro do caule (DC), altura da planta (AP), matéria seca da parte aérea (MSPA), potencial hidrogeniônico (pH) e Condutividade elétrica do extrato de saturação (CEes). A cobertura ajuda a minimizar os efeitos da irrigação com água de baixa e alta salinidade, para todas as variáveis analisadas. A água salina reduz o diâmetro do caule, mesmo na presença de cobertura morta. Os turnos de rega apresentam maiores valores de área foliar, altura de planta e diâmetro do caule, quando utilizados com cobertura morta.
\end{abstract}

Palavras-Chave: sorghum bicolor L., salinidade, cobertura do solo.

\section{SALT STRESS, DEAD COVERAGE AND WATER TURNING IN SORGO CULTURE}

\footnotetext{
ABSTRACT

${ }^{1}$ Graduanda em Agronomia, Instituto de Desenvolvimento Rural, Universidade da Integração Internacional da Lusofonia Afro-Brasileira, Redenção, Ceará, Brasil, ingryd.nojosal@gmail.com

2 Engenheira Agrônoma, Universidade da Integração Internacional da Lusofonia Afro-Brasileira, adilaagronomia@outlook.com

${ }^{3}$ Graduanda em Agronomia, Instituto de Desenvolvimento Rural, Universidade da Integração Internacional da Lusofonia Afro-Brasileira, Redenção, Ceará, Brasil, clarissamagalhaes.19@gmail.com

${ }^{4}$ Graduando em Agronomia, Instituto de Desenvolvimento Rural, Universidade da Integração Internacional da Lusofonia Afro-Brasileira, Redenção, Ceará, Brasil, thssousa2015@gmail.com

5 Professor Dr. Instituto de Desenvolvimento Rural, Universidade da Integração Internacional da Lusofonia AfroBrasileira, Redenção, Ceará, Brasil, sousagg@unilab.edu.br
} 
The objective of this work was to evaluate the effect of irrigation with high and low salinity water in different irrigation and mulch shifts on sorghum crop. The experiment was conducted in the experimental area of the Auroras Seedling Production Unit (UPMA) of the University of International Integration of Afro-Brazilian Lusophony (UNILAB), with a completely randomized design in a $2 \times 2 \times 2$ factorial arrangement, with two levels of electrical conductivity (A1 - water supply $0.8 \mathrm{dS} \mathrm{m}-1$ and A2 - saline $4.0 \mathrm{dS} \mathrm{m}-1$ ), two shifts. irrigation (T1 - daily watering shift and T2 - watering shift every 4 days) and two types of coverage ( $\mathrm{Cc}$ - with mulch and $\mathrm{Sc}$ - without mulch). The following variables were evaluated: leaf area (FA), stem diameter (DC), plant height (AP), shoot dry matter (MSPA), hydrogen potential $(\mathrm{pH})$ and saturation extract electrical conductivity (CEes). Coverage helps minimize the effects of low and high salinity water irrigation for all variables analyzed. Saline water reduces stem diameter even in the presence of mulch. Watering shifts have higher values of leaf area, plant height and stem diameter when used with mulch.

Keywords: sorghum bicolor L., salinity, groundcover.

\section{INTRODUÇÃO}

O Sorgo (Sorghum bicolor, L.) é uma grande fonte de energia, bastante utilizado pela sua adaptabilidade às regiões de seca (Nascimento et al., 2008), onde a produtividade de grãos e forragem não é muito alta (EMBRAPA, 2000). Entretanto, quando cultivada em condições de sequeiro, essa cultura tem sua produtividade afetada, em decorrência das flutuações pluviométricas, que se apresentam de maneira irregular durante o ciclo de desenvolvimento da cultura.

O semiárido brasileiro possui áreas com elevadas taxas de evapotranspiração maiores que os índices de precipitação. Devido essa característica, ocorre o acúmulo de sais na superfície dos solos, sendo necessário o aproveitamento de água salina. Paiva et al. (2016), consideram a existência elevada de teores de sais, nos mananciais de água subterrânea, estes provocam efeitos deletérios para germinação e produção agrícola. Ressalta-se que o estresse salino, promove o fechamento dos estômatos foliares e a redução na transpiração, e, consequentemente, diminuição na absorção de água e nutrientes pelas plantas (DIAS et al., 2017).

O uso da cobertura do solo, apresenta grande importância, devido esta possibilitar um maior tempo de retenção de água, fazendo assim com que este permaneça úmido. A cobertura do solo potencializa a produtividade das culturas (CARVALHO et al., 2018) e melhora os índices de produtividade da água (BRAGA et al., 2017)

Alencar et. al. (2009) declara o turno de rega como o intervalo compreendido entre duas irrigações sucessivas. O acúmulo de sais, ocasionados pelo estresse salino, pode vir a perturbar as funções fisiológicas e bioquímicas das plantas, fazendo com que ocorra alterações na absorção e nos nutrientes (AMORIM et. al., 2010), assim ocorrendo o retardamento do crescimento e minimizando a produção.

O objetivo deste trabalho foi avaliar o efeito da irrigação com água de alta e baixa salinidade em diferentes turnos de rega e cobertura morta na cultura do sorgo.

\section{MATERIAL E MÉTODOS}

O experimento foi realizado na área experimental da Unidade de Produção de Mudas Auroras (UPMA) da Universidade da Integração Internacional da Lusofonia AfroBrasileira (UNILAB), na região do Maciço de Baturité, na cidade de Redenção, no estado do Ceará, com coordenadas geográficas $4^{\circ} 13^{\prime} \mathrm{S}$, $38^{\circ} 43^{\prime} \mathrm{W}$, altitude média $92 \mathrm{~m}$. O experimento foi desenvolvido no período de novembro a dezembro de 2018.

O clima da região segundo a classificação Köppen é do tipo Aw', clima tropical com estação seca. O delineamento foi inteiramente casualizado, em arranjo fatorial 2 x 2 × 2, sendo dois níveis de condutividade 
elétrica (A1 - água de abastecimento $0,8 \mathrm{dS} \mathrm{m}^{-}$ ${ }^{1}$ e A2 - solução salina $4,0 \mathrm{dS} \mathrm{m}^{-1}$ ), dois turnos de rega ( $\mathrm{T} 1$ - turno de rega diário e $\mathrm{T} 2$ - turno de rega a cada 4 dias) e, dois tipos de cobertura $(\mathrm{Cc}$ - com cobertura morta e Sc sem cobertura). Foram utilizadas sementes de sorgo da variedade granífero, semeadas em vasos de material plástico flexível, com capacidade volumétrica de 11 litros, contendo substrato na proporção $3: 2: 1$, arisco, areia e matéria orgânica, respectivamente. As características químicas do substrato utilizado estão contidas na tabela 1 .

Tabela 1. Características químicas do substrato utilizado antes da aplicação dos tratamentos

\begin{tabular}{|c|c|c|c|c|c|c|c|c|}
\hline \multicolumn{9}{|c|}{ Características } \\
\hline M.O & $\mathrm{N}$ & $\mathrm{pH}$ & $\mathrm{P}$ & $\mathrm{K}$ & $\mathrm{Ca}$ & $\mathrm{Na}$ & PST & $\mathrm{CE}$ \\
\hline 7,3 & 0,41 & $\mathbf{7 , 8}$ & 45 & 2,07 & 2,2 & 1,04 & 14 & 2,35 \\
\hline & & - & $\mathrm{mg} \mathrm{Kg}^{-1}$ & & $\mathrm{I}_{c} \mathrm{dm}^{-3}$ & & $\%$ & $\mathrm{dS} \mathrm{m} \mathrm{m}^{-1}$ \\
\hline
\end{tabular}

Cada vaso foi semeado com seis sementes, após quatorze dias de semeadura foi realizado o desbaste manual, permanecendo apenas duas plantas por vaso, em seguida, foi realizada a aplicação do bagaço de cana-de-açúcar como cobertura e iniciou-se a irrigação com água salina em conjunto com os dois turnos de rega. No preparo da água de irrigação utilizaram-se os sais de $\mathrm{NaCl}, \mathrm{MgCl}_{2} .6 \mathrm{H}_{2} \mathrm{O} \mathrm{CaCl} 2.2 \mathrm{H}_{2} \mathrm{O}$ na proporção 7:2:1 conforme Medeiros (1992).

Após 38 dias da semeadura, foi realizado uma avaliação de crescimento, onde foram obtidos os valores de área foliar (AF), através da metodologia, estimada pelo método não destrutivo, (comprimento versus largura das folhas) multiplicando-se pelo fator de correção $(\mathrm{Fc}=0,75)$; diâmetro do caule (DC) utilizando-se um paquímetro digital com resultado expresso em milímetros; número de folhas (NF) por meio da contagem direta de folhas totalmente desenvolvidas e a altura da planta (AP), com o auxílio de uma régua graduada. Para a determinação de matéria seca da parte aérea (MSPA), as plantas foram acondicionadas em sacos de papel em ambiente protegido durante um período de 72 horas em estufa de circulação de ar, para que atingissem a massa constante. A pesagem foi realizada com o auxilio de uma balança de precisão.
Quanto ao solo, amostras de cada vaso foram utilizadas para determinar o $\mathrm{pH}$ e a condutividade elétrica do extrato de saturação do solo (CEes).

Os dados coletados foram submetidos à análise de variância (ANOVA), e os testes de médias pelo teste de Tukey ao nível de 5\% (*) e $1 \%(* *)$ de probabilidade, utilizando-se o programa computacional ASSISTAT. 7.6 Beta (SILVA; AZEVEDO, 2016).

\section{RESULTADOS E DISCUSSÃO}

Verificou-se a partir da análise de variância (Tabela 2) que houve interação significativa entre a salinidade da água de irrigação e a cobertura vegetal morta apenas para variável altura de plantas (AP) e diâmetro de caule (DC). Salinidade e turno de rega houve interação significativa para altura de planta (AP) e área foliar (AF).

Cobertura e turno de rega apresentou diferença significativa para todas as variáveis analisadas. $\mathrm{Na}$ interação tripla (salinidade $\mathrm{x}$ cobertura $x$ turno de rega) houve diferença significativa para altura de planta (AP), área foliar (AF), potencial hidrogeniônico $(\mathrm{pH})$ e condutividade elétrica do estrato de saturação (CEes). 
Tabela 2. Resumo da análise de variância pelo quadrado médio para as variáveis altura de plantas (AP), área foliar (AF), diâmetro do caule (DC), número de folhas (NF), massa seca da parte aérea (MSPSA), e massa seca da raiz (MSR) de plantas de sorgo em função de níveis de salinidade na água de irrigação sem e com cobertura vegetal morta e com turno de rega diário e Tr2 turno de rega a cada 3 dias.

\begin{tabular}{|c|c|c|c|c|c|c|c|}
\hline \multirow[b]{2}{*}{ FV } & \multirow[b]{2}{*}{ GL } & \multicolumn{6}{|c|}{ QUADRADO MÉDIO } \\
\hline & & AP & $\mathrm{AF}$ & DC & MSPA & $\mathrm{pH}$ & CEes \\
\hline Água (A) & 1 & $1394,28 * *$ & $26618,56 * *$ & $91,85 * *$ & $0,00001 \mathrm{~ns}$ & $0,16333 *$ & $7227664,08 * *$ \\
\hline Cobertura (C) & 1 & $804,42 * *$ & $16929,41 * *$ & $36,75^{* *}$ & $0,00012 \mathrm{~ns}$ & $0,00333 \mathrm{~ns}$ & $21505,33 \mathrm{~ns}$ \\
\hline Turno de rega $(\mathrm{T})$ & 1 & $148,75 \mathrm{~ns}$ & $9955,29 * *$ & $11,09 \mathrm{~ns}$ & $0,00004 \mathrm{~ns}$ & $0,00333 \mathrm{~ns}$ & $98826,75 \mathrm{~ns}$ \\
\hline $\mathrm{A} \times \mathrm{C}$ & 1 & $458,18 * *$ & $2228,09 n s$ & $34,71 * *$ & $0,00001 \mathrm{~ns}$ & $0,00083 \mathrm{~ns}$ & 221952,00ns \\
\hline $\mathrm{A} \times \mathrm{T}$ & 1 & $772,00 * *$ & $8773,66 * *$ & $6,27 \mathrm{~ns}$ & $0,00000 \mathrm{~ns}$ & $0,00750 \mathrm{~ns}$ & $34240,08 \mathrm{~ns}$ \\
\hline $\mathrm{C} \times \mathrm{T}$ & 1 & $693,88 * *$ & $8388,61 * *$ & $27,27 * *$ & $0,00031 *$ & $0,24083^{*}$ & $402600,33 *$ \\
\hline $\mathrm{A} \times \mathrm{C} \times \mathrm{T}$ & 1 & $1205,00 * *$ & $6865,75^{* *}$ & $7,87 \mathrm{~ns}$ & $0,00004 \mathrm{~ns}$ & $0,27000 *$ & $27,00 *$ \\
\hline Tratamentos & 7 & $782,36^{* *}$ & $11394,20 * *$ & $30,81 * *$ & $0,00007 \mathrm{~ns}$ & $0,09845^{*}$ & $1143830,79 * *$ \\
\hline Resíduo & 40 & 49,92 & 601,96 & 2,95 & 0,00007 & 0,03908 & 87652,25 \\
\hline C.V.\% & & 9,06 & 17,58 & 12,45 & 61,28 & 3,08 & 36,83 \\
\hline
\end{tabular}

FV: Fonte de variação; GL: Grau de liberdade; CV (\%): Coeficiente de variação; (**) significativo ao nível de $1 \%$ de probabilidade $(\mathrm{p}<.01) ;\left({ }^{*}\right)$ significativo ao nível de $5 \%$ de probabilidade $(.01=<\mathrm{p}<.05)$; (ns) não significativo $(\mathrm{p}>=$ $.05)$.

Os resultados apresentados na tabela 3 , são referentes a comparação de médias entre água, cobertura e turno de rega. Verifica-se que os valores dos tratamentos A1ScT2 E A2ScT2 não diferiram estatisticamente entre si, este último estatisticamente semelhante ao tratamento A2ScT1, sendo estes os menores resultados obtidos. Os tratamentos A2CcT1 e A2CcT2 também não diferiram estatisticamente entre si, bem como os tratamentos A1CcT1, A1CcT2 E A1ScT1, estes apresentando os melhores resultados.

O tratamento A1ScT1, apresentou valor superior ao A1CcT1, em relação a continuação de crescimento da área foliar, sem a presença de cobertura é uma demonstração de que a cultura começa a crescer na ausência da cobertura morta. Taiz e Zeiger (2009), relatam que plantas submetidas a estresses, como salino e hídrico, tendem a reduzir sua área foliar como mecanismo de defesa a fim de reduzir as perdas de água por transpiração. Mesmo sob condições de estresse, a cobertura reduziu as perdas de área foliar quando comparadas com aquelas sem cobertura, uma vez que uma maior proteção de solo confere menores perdas por evaporação, ocasionando melhor desenvolvimento da parte aérea (PEREIRA et al, 2015). Os dados de área foliar foram menores quando irrigados com a água salina, independentemente da cobertura e do turno de rega. Resultados semelhantes foram encontrados por Silva (2011), na cultura de feijão-de-corda, onde o aumento da salinidade inibiu afetou a área foliar. Essa inibição deve ter sido provocada, em maior parte, pelos efeitos tóxicos dos sais absorvidos pelas

Tabela 3. Comparação entre as médias de área foliar, em resposta à irrigação, turno de rega e cobertura.

\begin{tabular}{cccccc}
\hline \multirow{2}{*}{ Água } & \multicolumn{2}{c}{ Com Cobertura } & \multicolumn{2}{c}{ Sem Cobertura } & \multirow{2}{*}{ CV (\%) } \\
\cline { 2 - 4 } & \multicolumn{4}{c}{ Turno de rega } & \\
\hline & Diário & 4 dias & Diário & 4 dias & \\
\hline $0,8 \mathrm{dS} \mathrm{m}^{-1}$ & $177,825 \mathrm{aA}$ & $172,3417 \mathrm{aA}$ & $204,25 \mathrm{aA}$ & $98,0483 \mathrm{aB}$ & \multirow{2}{*}{17,58} \\
$4,0 \mathrm{dS} \mathrm{m}^{-1}$ & $141,2333 \mathrm{bA}$ & $141,99 \mathrm{bA}$ & $92,5667 \mathrm{bB}$ & $88,2833 \mathrm{aB}$ & \\
\hline
\end{tabular}

Letras maiúsculas na coluna e minúsculas linhas não diferem estatisticamente entre si pelo teste de tukey a $5 \%$ de probabilidade. 
Conforme apresentado na tabela 4, referente a comparação de médias entre água, cobertura e turno de rega. Verifica-se que os tratamentos A1CcT1, A1CcT2, A2CcT1 não diferiram estatisticamente entre si, o mesmo ocorreu com os tratamentos A1ScT2 e A2ScT2. Os tratamentos A2CcT2 e A1ScT1 são semelhantes entre si. E o tratamento A2ScT1 apresentou menor valor, mas não difere entre si, quando comparado aos tratamentos A1ScT2 e A2ScT2. De forma semelhante, no que concerne à altura de plantas, o uso da cobertura morta atenuou os efeitos deletérios causados pela salinidade da água de irrigação quando comparados ao não uso de coberturas, de forma que o turno de rega diário com água salina nesta situação contribuiu negativamente com a altura do sorgo.

A redução do potencial osmótico do solo causado pelos sais solúveis presentes prejudica os processos fisiológicos das culturas, como a atividade meristemática e o alongamento celular devido à redução na absorção de água pelas raízes (AYERS E WESTCOT,1999).

Tabela 4. Comparação entre as médias de altura de plantas da cultura do sorgo, em resposta à irrigação, turno de rega e cobertura.

\begin{tabular}{cccccc}
\hline \multirow{2}{*}{ Água } & \multicolumn{3}{c}{ Com Cobertura } & \multirow{2}{*}{ CV (\%) } \\
\cline { 2 - 5 } & \multicolumn{4}{c}{ Turno de rega } & \\
\hline & Diário & 4 dias & Diário & 4 dias \\
\hline $0,8 \mathrm{dS} \mathrm{m}^{-1}$ & $81,3833 \mathrm{aB}$ & $87,4667 \mathrm{aAB}$ & $97 \mathrm{aA}$ & $67,8333 \mathrm{aC}$ & \multirow{2}{*}{9,06} \\
$4,0 \mathrm{dS} \mathrm{m}^{-1}$ & $78,7833 \mathrm{aAB}$ & $80,8667 \mathrm{aA}$ & $62 \mathrm{bC}$ & $68,9167 \mathrm{aBC}$ & \\
\hline
\end{tabular}

Letras maiúsculas na coluna e minúsculas linhas não diferem estatisticamente entre si pelo teste de tukey a $5 \%$ de probabilidade.

Como apresentado na tabela 5, referente a comparação de médias entre a água de irrigação e cobertura para o diâmetro do caule, os tratamentos $\mathrm{A} 1 \mathrm{Cc}, \mathrm{A} 1 \mathrm{Sc}$ e $\mathrm{A} 2 \mathrm{Cc}$ não diferiram estatisticamente entre si. $\mathrm{O}$ tratamento A2Sc foi o único que apresentou diferença estatística entre os demais tratamentos, este apresentando o menor valor obtido. Da mesma forma, a cobertura morta influenciou a redução dos efeitos prejudiciais da água salina na cultura do sorgo onde, para a mesma água aplicada, os valores de diâmetro do caule de plantas que não possuíam cobertura no solo foram inferiores aos demais. Salienta-se que o crescimento vegetal pode sofrer restrição caso elementos minerais, como o sódio, estão presentes no solo e atinjam níveis que limitem a disponibilidade hídrica ou excedam a faixa adequada de um outro nutriente (Taiz \& Zeiger 2009). Além disso, o uso de cobertura morta, como proteção do solo, diminui a evaporação da água adicionada durante a irrigação, evitando a precipitação de sais na zona radicular (COSTA et al., 2008).

Tabela 5. Comparação entre as médias de diâmetro do caule da cultura do sorgo, em resposta à irrigação e cobertura.

\begin{tabular}{ccc}
\hline \multirow{2}{*}{ Água } & \multicolumn{2}{c}{ Cobertura } \\
\cline { 2 - 3 } & Com Cobertura & Sem Cobertura \\
\hline $0,8 \mathrm{dS} \mathrm{m}^{-1}$ & $15,2283 \mathrm{aA}$ & $15,1792 \mathrm{aA}$ \\
$4,0 \mathrm{dS} \mathrm{m}^{-1}$ & $14,1625 \mathrm{aA}$ & $10,7117 \mathrm{bB}$ \\
\hline
\end{tabular}

Letras maiúsculas na coluna e minúsculas linhas não diferem estatisticamente entre si pelo teste de tukey a 5\% de probabilidade.

Em relação a comparação de médias entre cobertura e o turno de rega para o diâmetro do caule, apresentada na tabela 6, o tratamento ScT2 apresentou diferença significativa entre os demais tratamentos, CcT1, CcT2, ScT1, estes não diferindo 
estatisticamente entre si. No que concerne ainda ao diâmetro do caule, o uso de cobertura contribuiu para a regulação da evapotranspiração da cultura pela retenção de umidade no solo (VIANA et al., 2012), permitindo que esta se desenvolvesse normalmente e que, mesmo irrigada com águas salinas, obtivesse resultados semelhantes quando comparada ao ser irrigada com água não salina, não diferindo estatisticamente quanto ao turno de rega dentro da água 1, tendo os piores resultados expressos quando utilizada a água salina combinada ao não uso de cobertura.

Tabela 6. Comparação entre as médias de diâmetro do caule da cultura do sorgo, em resposta à cobertura e turno de rega.

\begin{tabular}{ccc}
\hline \multirow{2}{*}{ Cobertura } & \multicolumn{2}{c}{ Turno de Rega } \\
\cline { 2 - 3 } & Diário & 4 dias \\
\hline Com Cobertura & $14,4225 \mathrm{aA}$ & $14,9683 \mathrm{aA}$ \\
Sem Cobertura & $14,18 \mathrm{aA}$ & $11,7108 \mathrm{bB}$ \\
\hline
\end{tabular}

Letras maiúsculas na coluna e minúsculas linhas não diferem estatisticamente entre si pelo teste de tukey a $5 \%$ de probabilidade.

Conforme apresentado na tabela 7 , a matéria seca da parte aérea teve seus resultados reduzidos quando na ausência de cobertura morta no solo num intervalo de irrigação de 4 dias, embora o turno de rega não tenha influenciado nessa redução, de forma que os tratamentos com cobertura não diferiram estatisticamente, nem o tratamento sem cobertura com irrigação diária.

Tabela 7. Comparação entre as médias de matéria seca da parte aérea da cultura do sorgo em resposta à cobertura e turno de rega.

\begin{tabular}{ccc}
\hline \multirow{2}{*}{ Cobertura } & \multicolumn{2}{c}{ Turno de Rega } \\
\cline { 2 - 3 } & Diário & 4 dias \\
\hline Com Cobertura & $0,0138 \mathrm{aA}$ & $0,0171 \mathrm{aA}$ \\
Sem Cobertura & $0,0157 \mathrm{aA}$ & $0,0088 \mathrm{bA}$
\end{tabular}

Letras maiúsculas na coluna e minúsculas linhas não diferem estatisticamente entre si pelo teste de tukey a $5 \%$ de probabilidade.

Essa redução na MSPA da cultura na ausência da cobertura morta está relacionada com o maior custo energético utilizado pela cultura para que se mantenha viva em virtude da redução da água disponível no solo sem cobertura, fazendo com que a cultura invista mais na sua sobrevivência do que no acúmulo dos fotoassimilados (TAIZ et al., 2017). Sousa et al. (2018) verificaram redução da massa seca da parte aérea em plantas de milho na ausência de cobertura morta no solo. Como apresentado na tabela 8, os solos irrigados com água salina obtiveram resultados de $\mathrm{pH}$ mais elevados do que os irrigados com a água de abastecimento, porém demonstrando resultados inferiores ao $\mathrm{pH}$ original do solo em ambos os casos $(7,8)$, não diferindo estatisticamente quando referente às coberturas e turnos de rega. 
Tabela 8. Potencial hidrogeniônico do solo cultivado com sorgo sob estresse salino e diferentes turnos de rega na presença e ausência de cobertura morta.

\begin{tabular}{cccccc}
\hline \multirow{2}{*}{ Água } & \multicolumn{3}{c}{ Com Cobertura } & \multicolumn{2}{c}{ Sem Cobertura } \\
\cline { 2 - 5 } & \multicolumn{4}{c}{ Turno de rega } \\
\hline & Diário & 4 dias & Diário & 4 dias \\
\cline { 2 - 5 } $0,8 \mathrm{dS} \mathrm{m}^{-1}$ & $6,2333 \mathrm{bAB}$ & $6,4833 \mathrm{aAB}$ & $6,5167 \mathrm{aA}$ & $6,1833 \mathrm{bB}$ \\
$4,0 \mathrm{dS} \mathrm{m}^{-1}$ & $6,4833 \mathrm{aA}$ & $6,4833 \mathrm{aA}$ & $6,4500 \mathrm{aA}$ & $6,4667 \mathrm{aA}$ & 10,09 \\
\hline
\end{tabular}

Letras maiúsculas na coluna e minúsculas linhas não diferem estatisticamente entre si pelo teste de tukey a $5 \%$ de probabilidade.

Estes resultados demonstram que, independente da água utilizada, os sais presentes tendem a reduzir o $\mathrm{pH}$ original do substrato (CAVALCANTE et al., 2010), tendo seus efeitos menos severos na água mais salina. Em contrapartida, Sousa et al. (2012) verificaram que solos cultivados com amendoim e irrigados com águas salinas diminuíram linearmente o $\mathrm{pH}$ do solo com o aumento da condutividade elétrica da água de irrigação.
$\mathrm{Na}$ Tabela 9 verifica-se que o extrato de saturação do solo, em relação à sua CEes original, sofreu redução em todos os tratamentos aplicados, mas quando utilizada a água salina essa redução foi mais atenuada, independentemente da presença da cobertura ou turno de rega, não diferindo estatisticamente entre si, enquanto que os tratamentos com água de abastecimento obtiveram os menores resultados, não diferindo estatisticamente entre si.

Tabela 9. Condutividade elétrica do extrato de saturação do solo (CEes) cultivado com sorgo sob estresse salino e diferentes turnos de rega na presença e ausência de cobertura morta.

\begin{tabular}{|c|c|c|c|c|}
\hline \multirow{3}{*}{ Água } & \multicolumn{2}{|c|}{ Com Cobertura } & \multicolumn{2}{|c|}{ Sem Cobertura } \\
\hline & \multicolumn{4}{|c|}{ Turno de rega } \\
\hline & Diário & 4 dias & Diário & 4 dias \\
\hline $0,8 \mathrm{dS} \mathrm{m}^{-1}$ & $389,3333 \mathrm{bA}$ & $348,8333 \mathrm{bA}$ & 298,3333 bA & $627,1667 \mathrm{bA}$ \\
\hline $4,0 \mathrm{dS} \mathrm{m}^{-1}$ & $1353,3330 \mathrm{aA}$ & $1209,0000 \mathrm{aA}$ & $993,3333 \mathrm{aA}$ & $1212,3330 \mathrm{aA}$ \\
\hline
\end{tabular}

Letras maiúsculas na coluna e minúsculas linhas não diferem estatisticamente entre si pelo teste de tukey a 5\% de probabilidade.

Possivelmente a quantidade de água aplicada nos tratamentos foi o suficiente para reduzir a quantidade de sais presentes no substrato original, atuando como uma fração de lixiviação para estes sais.

Resultados semelhantes foram obtidos por Silva et al. (2011), em que verificaram que o conteúdo salino do solo cultivado com feijão-caupi aumentou significativamente com o teor salino das águas. Comportamento similar foi observado por Lima Neto et al. (2018) trabalhando com salinidade nas mudas de tamarindo, constataram crescimento linear na CEes à medida que aumentava a concentração salina.

\section{CONCLUSÕES}

A cobertura ajuda a minimizar os efeitos da irrigação com água de baixa e alta salinidade, para todas as variáveis analisadas. A água salina $\left(4,0 \mathrm{dS} \mathrm{m}^{-1}\right)$ reduz o diâmetro do caule, mesmo na presença de cobertura morta. Os turnos de rega apresentam maiores valores de área foliar, altura de planta e diâmetro do caule, quando utilizados com cobertura morta. A água de 4,0 $\mathrm{dS} \mathrm{m}^{-1}$ aliada ao seu uso em solos sem cobertura e/ou aplicadas em intervalos de irrigação maiores, reduzem a matéria seca da parte aérea da cultura do sorgo. A água de baixa salinidade $\left(0,8 \mathrm{dS} \mathrm{m}^{-1}\right)$ 
aplicada ao solo cultivado com sorgo reduz a condutividade elétrica do extrato de saturação.

\section{AGRADECIMENTOS}

À Fundação Cearense de Apoio ao Desenvolvimento Científico e Tecnológico (FUNCAP) pelo apoio financeiro.

\section{REFERÊNCIAS BIBLIOGRÁFICAS}

ALENCAR, C. A. B. de; CUNHA, F. F. da; MARTINS, C. E; CÓSER, A. C; ROCHA, W. S. D. da; ARAÚJO, R. A. S. Irrigação de pastagem: atualidade e recomendações para uso e manejo. Revista brasileira de zootecnia, v. 38, (número especial), p. 98-108, $2009 . \quad$ https://doi.org/10.1590/S151635982009001300012.

AMORIM, A. V.; GOMES FILHO, E.; BEZERRA, M. A.; PRISCO, J. T.; LACERDA, C. F. Respostas fisiológicas de plantas adultas de cajueiro anão precoce à salinidade. Revista Ciência Agronômica, v. $41, \quad$ n. $1, \quad$ p. 113-121, 2010. https://doi.org/10.5935/1806-6690.20100016.

AYERS, R. S.; WESTCOT, D. W. A qualidade da água na agricultura. 2 ed. Campina Grande: UFPB. p. 218, 1999.

BRAGA, M. B.; MAROUELLI, W. A.; RESENDE, G. M.; MOURA, M. S. B.; COSTA, N. D.; CALGARO, M.; CORREIA, J. S. Coberturas do solo e uso de manta agrotêxtil (TNT) no cultivo do meloeiro. Horticultura Brasileira, v. 35, n. 1, p. 147153, 2017. http://dx.doi.org/10.1590/S0102053620170123.

CARVALHO, D. F. de; RIBEIRO, E. C.; GOMES, D. P. Marketable yield of onion under different irrigation depths, with and without mulch. Revista Brasileira de Engenharia Agrícola e Ambiental, v. 22, n. 2, p. 107-112, 2018. https://doi.org/10.1590/1807-

1929/agriambi.v22n2p107-112.

CAVALCANTE, L. F.; CORDEIRO, J. C.; NASCIMENTO, J. A. M.; CAVALCANTE, I. H. L.; DIAS, T. J. Fontes e níveis da salinidade da água na formação de mudas de mamoeiro cv. Sunrise solo. Semina, v.31, suplemento 1, p.1281- 1290, 2010. http://dx.doi.org/10.5433/1679-

0359.2010v31n4Sup1p1281.

COSTA, D. M. A. da; MELO, H. N. de SOUZA; FERREIRA, S. R.; HOLANDA, J. S. de. Crescimento e desenvolvimento do Amaranto (Amaranthus spp.) sob estresse salino e cobertura morta. Revista Brasileira de Ciência do Solo, v. 32, n. 1, p. 43-48, $2008 . \quad$ https://doi.org/10.1590/S010006832008000100005.

DIAS, A. S.; LIMA, G. S.; GHEYI, H. R.; SOARES, L. A. A.; SOUZA, L. P.; BEZERRA, I. L. Crescimento do algodoeiro 'brs rubi' em função da irrigação com águas salinas e adubação nitrogenada. Revista Brasileira de Agricultura Irrigada v.11, n. 7, p. $1945 \quad$ - $1955,2017$. https://doi.org/10.7127/rbai.v11n700660.

LIMA NETO, A. J.; CAVALCANTE, L. F.; NUNES, J. C.; SOUTO, A. G. de L.; BEZERRA, F. T. C.; Mudas de tamarindeiro irrigadas com água salina em solo sem e com biofertilizantes. Irriga, v. 20, n. 4, p. 730-744, 2018. https://doi.org/10.15809/irriga.2015v20n4p73 0 .

MEDEIROS, J. F. Qualidade da água de irrigação utilizada nas propriedades assistidas pelo "GAT" nos Estados do RN, PB, CE e avaliação da salinidade dos solos. 1992. 173 f. Dissertação (Mestrado em Engenharia Agrícola) - Universidade Federal da Paraíba, Campina Grande.

NASCIMENTO, W. G.; PRADO, I. N.; JOBIM, C. C.; EMILE, J. C.; SURAULT, F.; HUYGHE, C. Valor alimentício das silagens 
de milho e de sorgo e sua influência no desempenho de vacas leiteiras. Revista Brasileira de Zootecnia, v. 37, n. 5, p. 896904, 2008. http://dx.doi.org/10.1590/S151635982008000500018 .

PAIVA, F. I. G.; GURGEL, M. T.; OLIVEIRA, F. A.; MOTA, A. F.; COSTA, L. R. \& OLIVEIRA JUNIOR, H. S. Qualidade da fíbra do algodoeiro BRS Verde irrigado com água de diferentes níveis salinos. Irriga, vol. 1, n. 1, p. 209-220, 2016. https://doi.org/10.15809/irriga.2016v1n1p209220

PEREIRA, F. F. S.; MATSURA, E. E.; MOUSINHO, F. E. P.; BIZARI, D. R. Retenção de água em níveis de cobertura morta no feijoeiro irrigado em sistema de plantio direto. Irriga, v. 20, n. 3, p. 557-569, 2015.

https://doi.org/10.15809/irriga.2015v20n3p55 7.

RIBAS, Paulo Motta. EMBRAPA Milho e Sorgo Sistema de Produção, 2. EMBRAPA, 2000 .

SILVA, F. A. S.; AZEVEDO, C. A. V. The Assistat Software Version 7.7 and its use in the analysis of experimental data. Africa Journal and Agriculture Researche, v. 11, n. 39 , p.3733-3740, 2016. DOI: 10.5897/AJAR2016.11522.

SILVA, F. L. B.; LACERDA, C. F.; SOUSA, G. G.; NEVES, A. L. R.; SILVA, G. L.; SOUSA, C. H. C. Interação entre salinidade e biofertilizante bovino na cultura do feijão-decorda. Revista Brasileira de Engenharia
Agrícola e Ambiental, v. 15, n. 4, p. $383-$ 389 , 2011. DOI: $10.1590 / \mathrm{S} 1415-$ 43662011000400009

SOUSA, G. G.; AZEVEDO, B. M.; ALBUQUERQUE, A. H. P.; MESQUITA, J. B. R.; VIANA, T. V. A. Características agronômicas do amendoinzeiro sob irrigação com águas salinas em solo com biofertilizantes. Revista Agro@mbiente Online, v. 6, n. 2, p. 124-132, 2012. http://dx.doi.org/10.18227/1982-

8470ragro.v6i2.708.

SOUSA, G. G.; RODRIGUES, V. S.; SALES, J. R. S.; CAVALCANTE, F.; SILVA, G. L.; LEITE, K. N. Estresse salino e cobertura vegetal morta na cultura do milho. Revista Brasileira de Agricultura Irrigada, v. 12, n. 7, p. $3078-3089, \quad 2018 . \quad$ DOI: 10.7127/rbai.v12n700889.

Taiz, L.; Zeiger, E. Plant physiology. 4nd ed. Sunderland: Sinauer Associates, Inc. Publishers, 2009. 848p.

TAIZ, L.; ZEIGER, E.; MOLLER, I.; MURPHY, A. Fisiologia e Desenvolvimento Vegetal. 6. ed. - Porto Alegre: Artmed, 2017.

VIANA, T. V. de A.; LIMA, A. D.; MARINHO, A. B.; DUARTE, J. M. de L.; AZEVEDO, B. M. de; COSTA, S. C. Lâminas de irrigação e coberturas do solo na cultura do girassol, sob condições semiáridas. Irriga, v. $17, \quad$ n. 2, p. 126-136, 2012. https://doi.org/10.15809/irriga.2012v17n2p12 6. 